\section{Staphylococcus lugdunensis: novel organism causing cochlear implant infection}

\author{
Samina Bhumbra, Mona Mahboubi, \\ R. Alexander Blackwood
}

Division of Pediatric Infectious Diseases, University of Michigan, Ann Arbor, MI, USA

\begin{abstract}
A majority of cochlear implant infections are caused by Staphylococcus aureus or Pseudomonas aeruginosa. Reported here is a pediatric patient with a cochlear implant infection caused by methicillin-resistant Staphylococcus lugdunensis, a coagulase-negative Staphylococcus that has only recently been determined to be clinically relevant (1988). Unlike other coagulase-negative Staphylococcus, it is more aggressive, carrying a greater potential for tissue destruction. In pediatrics, the organism is uncommon, poorly described, and generally pan-susceptible. Described herein is the presentation and management of this unusual organism in a pediatric setting.
\end{abstract}

\section{Case Report}

A 6-year-old female with bilateral congenital deafness status post right Nucleus Freedom internal cochlear implant in 2011 presented to ENT clinic with a one week history of right posterior auricular swelling over the implant transmitter site. The examination was notable for local tenderness only without fever, erythema, or any other systemic symptoms. The patient was initiated on a course of cephalexin. Two days later, the area in question began to drain spontaneously, and the patient was admitted for further evaluation. On physical examination, the patient's temperature was $36.6^{\circ} \mathrm{C}$, pulse 107 , respiration 20 , blood pressure $110 / 74$, with oxygen saturation of $98 \%$ on room air. Significant physical findings included pinpoint area on the right posterior auricular skin overlying the implant and expressing thick white-yellowish fluid. There was mild tenderness to palpation but no evidence of overlying erythema or induration. Cultures from the wound and drainage were obtained and cephalexin was broadened to piperacillin/tazobactam and cefuroxime. Laboratory work was as follows: white blood cell count $10.2 \mathrm{~K} / \mathrm{cm}^{3}$ with $39.1 \%$ neutrophils and $52.4 \%$ lymphocytes, hemoglobin $12.7 \mathrm{~g} / \mathrm{dL}$, platelets $333 \mathrm{~K} / \mathrm{cm}^{3}$, C-reactive protein $<0.1$ $\mathrm{mg} / \mathrm{dL}$, and erythrocyte sedimentation rate 6 $\mathrm{mm}$. The patient was taken to the operating room for exploration and drainage. Intraoperative findings revealed that the cochlear transplant transmitter was encased in a gelatinous material and further cultures were obtained. All cultures grew gram-positive cocci in clusters and subsequently were identified as Staphylococcus lugdunensis (MALDITOF; Bruker Daltonics, Bellerica, MA, USA) resistant (by VITEK 2; bioMérieux, Durham, NC, USA) to methicillin, but susceptive to clindamycin, vancomycin, trimethoprim/sulfamethoxazole, erythromycin, and tetracycline. Pediatric Infectious Diseases division was consulted, and patient was discharged home on oral trimethoprim/sulfamethoxazole with scheduled follow-up in three weeks because the family desired an attempt be made to salvage the implant. Following discharge, the surgical incision site along the right posterior auricular area continued to drain purulent and malodorous fluid. The patient remained afebrile without any other systemic symptoms. Seventeen days after discharge, the patient was re-evaluated by ENT, was noted to have a dehiscent, gaping wound with a crust covering and an exposed implant. The patient was taken back to the operating room for debridement and closure of the right posterior auricular wound. Gelatinous material was found tracking along the electrodes into the mastoid cavity. The gelatinous material was removed and the wound was irrigated with betadine, gentamicin solution, and bacitracin solutions. Cultures from the procedure grew $S$. lugdunensis with the same susceptibility profile as previous cultures. Eleven days following this second surgical procedure, drainage was noted along the incision site. The patient was taken to the operating room a third time, and the cochlear implant was removed. Finalized cultures obtained from the tissue cochlear implant grew $S$. lugdunensis, 3 morphotypes of Viridians group Streptococcus, and a rare quantity of Actinomyces species resembling Actinomyces graevenitzii. The patient continued on trimethoprim/sulfamethoxazole for an additional two-week course from the cochlear implant removal. Upon the completion of this two-week course, Pediatric Infectious Diseases evaluated the patient, and the incision site was healing well with no further drainage noted. To the knowledge of these authors, the patient has had no further infectious issues with respect to this cochlear implant infection.

\section{Discussion}

Staphylococcus lugdunensis is a coagulase
Correspondence: Roland Alexander Blackwood, Division of Pediatric Infectious Diseases, University of Michigan, D5101 Medical Professional Building, 1500 East Medical Center Drive SPC 5718, Ann Arbor, MI 48109, USA. Tel.: +1.734.763.2440 - Fax: +1.734.232.3859. E-mail: rab@med.umich.edu

Key words: Staphylococcus lugdunensis, cochlear implant, coagulase negative staphylococcus.

Contributions: SB, wrote the initial draft of the manuscript, in particular the abstract and the case report; MM, edited the abstract and case report and wrote the discussion; RAB edited and coordinated the manuscript.

Conflict of interests: the authors declare no potential conflict of interests.

Received for publication: 26 March 2014.

Revision received: 30 April 2014

Accepted for publication: 7 May 2014.

This work is licensed under a Creative Commons Attribution NonCommercial 3.0 License (CC BYNC 3.0).

CCopyright S. Bhumbra et al., 2014 Licensee PAGEPress, Italy

Infectious Disease Reports 2014; 6:5406

doi:10.4081/idr.2014.5406

negative Staphylococcus first described by Freney et al. in 1988. ${ }^{1}$ Coagulase negative Staphylococcus (CoNS) is considered normal skin flora. ${ }^{2}$ However, in context of medical devices, catheters, and other foreign body instrumentation, colonization CoNS may be pathogenic. While CoNS is typically associated with less severe and sub-acute illnesses, $S$. lugdunensis is potentially more virulent than other CoNS and has been associated with endocarditis, CNS infections, peritonitis, bacteremia, osteomyelitis, skin and soft tissue abscesses, infections of prostheses, and surgical site infections. ${ }^{3,4} S$. lugdunensis potential virulence is similar $S$. aureus. Of note, the microbiology laboratory typically has a twostep process for identification of organisms as either Staphylococcus aureus (coagulase positive Staphylococcus) or CoNS: a slide test and confirmatory tube test. Often for S. lugdunen$s i s$, its clumping factor results in a positive slide agglutination test that is similarly seen with Staphylococcus aureus. ${ }^{3}$

Typically $S$. lugdunensis is susceptible to methicillin; however resistant isolates have been emerging, first described in China, Brazil, and Europe. ${ }^{5}$ Current literature on $S$. lugdunensis is primarily limited to adult patients; pediatric cases have been reported with VP shunt infections and endocarditis. ${ }^{6,7}$ The majority of the isolates have been methi- 
cillin susceptible. The emergence of resistance to methicillin has appeared internationally including in a 29-week gestation neonate with sepsis located in Singapore, but there have been limited reports in the United States. ${ }^{8}$ To the author's knowledge, there has been only one U.S. reported case of methicillin resistant $S$. lugdunensis, and this was in a pediatric patient with endocarditis. ${ }^{7}$

Cochlear implantation is a complex, invasive surgical procedure; however, the rate of surgical complications is reasonably low with the most common complication being postoperative infection of the skin and soft tissue. Causes for these infections vary but share the common risk of hematogenous seeding and the potential for meningitis. Davids et al. reviewed 462 patients, and found that two (0.43\%) patients developed infections. One was the result of picking at the skin over the implant, and the second case was caused by a hematoma becoming infected on postoperative day 1 . Both required surgical intervention. $S$. aureus was identified in the first implant while the other remained unidentified by the authors. ${ }^{9}$ Comparatively, Loundon et al. reported 15 of 434 (3.5\%) of pediatric patients presented with cutaneous infections requiring surgical intervention of the cochlear implant with a mean delay of 2.2 years. Although organisms were not specified in the report, the authors noted that recurrent infections among the major soft tissue infections were due to biofilm. ${ }^{10}$ Looking back at older reviews, the complication rate from 1990 to 2007 was higher at $6.8 \%$. The culture results of the infections were published, and the most common organisms were $S$. aureus and Pseudomonas aeruginosa. Other organisms that were identified by culture included in decreasing order: CoNS, Alcaligenes xylosoxidans, group $\mathbf{G}$ Streptococcus, Candida albicans, and diphtheroids. None of these organisms showed unusual antimicrobial resistance patterns. ${ }^{11}$

There are several aspects that make this case presentation unique. First, the patient was complication free for two years following placement of the implant. The presentation was unusual because there was no evidence of trauma, induration, or erythema over the area of infection with no previous history of antibiotic use. Additionally, the patient presented with a methicillin-resistant isolate of $S$. lugdunensis. Based on literature review, this is the first reported case of methicillin-resistant $S$. lugdunensis cochlear implant infection in the pediatric population. Only one other report available describes similar $S$. lugdunensis infections on foreign bodies in adults with 4 of 28 isolates being methicillin-resistant. ${ }^{12}$ In this study, the authors emphasized the similarities between $S$. aureus and $S$. lugdunensis, which altered the treatment protocol usually used for CoNS. For example, a minimum inhibitory concentration (MIC) of methicillin $\geq 1 \mu \mathrm{g} / \mathrm{mL}$ would typically exclude its use in treating CoNS other than $S$. lugdunensis; however, this same MIC is considered susceptible for $S$. lugdunensis. Of note, the authors recognized that ß-lactams offered better penetration into bone versus vancomycin. This difference in acceptable MIC resulted in the vancomycin group returning sooner with an infection compared to the B-lactam group.

Treatment of $S$. lugdunensis in our patient has proven challenging because a biofilm organized around the implant unit based on the surgical reports. It has been noted that the pathogenesis of $S$. lugdunensis includes the production of glycocalyx as a virulence factor in $25 \%$ of cases. ${ }^{13}$ Although our patient was placed on the appropriate antibiotic therapy, the poor vascularization, as well as the presence of a biofilm, probably prevented the trimethoprim/sulfamethoxazole from effectively penetrating the area of infection. A similar case was reported involving an infected cochlear implant several years post placement that developed a biofilm formed by methicillin sensitive $S$. aureus. Despite combination therapy including rifampin to penetrate the biofilm, removal of the implant was also required for definitive treatment. ${ }^{14}$

\section{Conclusions}

The importance of differentiating CoNS at the species level at times is critical for treatment. In the past, most laboratories would not routinely identify CoNS to a species level. However, with advancements in microlaboratory capabilities to identify CoNS to the species level, identification at species level can be routinely achieved. ${ }^{15}$ Our current case illustrates the difficulty in eradicating $S$. lugdunensis with the foreign material in place despite appropriate antibiotics making a strong argument for early removal in similar situations.

\section{References}

1. Freney J, Brun Y, Bes $M$, et al. Staphylococcus lugdunensis sp. nov. and Staphylococcus schleiferi sp. nov., two species from human clinical specimens. Int J System Bacteriol 1988;38:168-72.
2. Bieber L, Kahlmeter G. Staphylococcus lugdunensis in several niches of the normal skin flora. Clin Microbiol Infect 2010;16:385-8.

3. Frank KL, Del Pozo JL, Patel R. From clinical microbiology to infection pathogenesis: how daring to be different works for Staphylococcus lugdunensis. Clin Microbiol Rev 2008;21:111-33.

4. Ravaioli S, Selan L, Visai L, et al. Staphylococcus lugdunensis, an aggressive coagulase-negative pathogen not to be underestimated. Int $\mathrm{J}$ Artif Organs 2012; 35:742-53.

5. Babu E, Oropello J. Staphylococcus lugdunensis: the coagulase-negative staphylococcus you don't want to ignore. Expert Rev Anti Infect Ther 2011;9:901-7.

6. Elliott SP, Yogev R, Shulman ST. Staphylococcus lugdunensis: an emerging cause of ventriculoperitoneal shunt infections. Pediatr Neurosurg 2001;35:128-30.

7. Murillo J, McMahon C, Starr J. Large vegetations in Staphylococcus lugdunensis endocarditis. Heart Lung 2008;37:479-80.

8. Tee WS, Soh SY, Lin R, Loo LH. Staphylococcus lugdunensis carrying the mecA gene causes catheter-associated bloodstream infection in premature neonate. J Clin Microbiol 2003;41:519-20.

9. Davids T, Ramsden JD, Gordon KA, et al. Soft tissue complications after small incision pediatric cochlear implantation. Laryngoscope 2009;119:980-3.

10. Loundon N, Blanchard M, Roger G, et al. Medical and surgical complications in pediatric cochlear implantation. Arch Otolaryngol Head Neck Surg 2010;136:125 .

11. Hopfenspirger MT, Levine SC, Rimell FL. Infectious complications in pediatric cochlear implants. Laryngoscope 2007;117: 1825-9.

12. Shah NB, Osmon DR, Fadel H, et al. Laboratory and clinical characteristics of Staphylococcus lugdunensis prosthetic joint infections. J Clin Microbiol 2010;48: 1600-3.

13. von Eiff C, Peters G, Heilmann C. Pathogenesis of infections due to coagulase-negative staphylococci. Lancet Infect Dis 2002;2:677-85.

14. Vaid N, Vaid S, Manikoth M. Case report biofilm infection of a cochlear implant. Cochlear Implants Int 2013;14:117-20.

15. Harris LG, El-Bouri K, Johnston S, et al. Rapid identification of staphylococci from prosthetic joint infections using MALDITOF mass-spectrometry. Int J Artif Organs 2010;33:568-74. 